OPEN ACCESS

Edited by:

Mario U. Manto,

University of Mons, Belgium

Reviewed by:

Elise Lesage,

Ghent University, Belgium

De-Lai Qiu,

Yanbian University, China

*Correspondence:

Yi-Shin Sheu

yishin.sheu@gmail.com

John E. Desmond

dr.jdesmond@gmail.com

Received: 30 October 2018 Accepted: 04 February 2019 Published: 21 February 2019

Citation:

Sheu Y-S, Liang Y and Desmond JE (2019) Disruption of Cerebellar Prediction in Verbal Working Memory.

Front. Hum. Neurosci. 13:61. doi: 10.3389/fnhum.2019.00061

\section{Disruption of Cerebellar Prediction in Verbal Working Memory}

\author{
Yi-Shin Sheu*, Yu Liang and John E. Desmond* \\ Department of Neurology, Division of Cognitive Neuroscience, Johns Hopkins University School of Medicine, Baltimore, MD, \\ United States
}

Mounting evidence suggests that the right cerebellum contributes to verbal working memory, but the functional role of this contribution remains unclear. In an established theory of motor control, the cerebellum is thought to predict sensory consequences of movements through an internal "forward model." Here, we hypothesize a similar predictive process can generalize to cerebellar non-motor function, and that the right cerebellum plays a predictive role that is beneficial for rapidly engaging the phonological loop in verbal working memory. To test this hypothesis, double-pulse transcranial magnetic stimulation (TMS) was administered over either the right cerebellum or right occipital lobe (control site), on half the trials, to interrupt the rehearsal of a 6-letter sequence. We found that cerebellar stimulation resulted in greater errors in participants' report of the letter in the current position. Additional analyses revealed that immediately after cerebellar TMS, participants were more likely to use out of date information to predict the next letter in the sequence. This pattern of errors is consistent with TMS causing a temporary disruption of state estimation and cerebellar forward model function, leading to prediction errors in the phonological loop.

Keywords: cerebellum, TMS, verbal working memory, forward models, prediction

\section{INTRODUCTION}

It has become widely accepted in recent years that the human cerebellum contributes not only to motor function, but also to a wide range of non-motor cognitive functions (for reviews, see Stoodley, 2012; Buckner, 2013; Schmahmann, 2019), such as verbal working memory (Chein and Fiez, 2001; Chen and Desmond, 2005; Justus et al., 2005; Ravizza et al., 2006; Hayter et al., 2007; Durisko and Fiez, 2010; Marvel and Desmond, 2010; Peterburs et al., 2010, 2016; Stoodley et al., 2012), executive function (Grafman et al., 1992; Rao et al., 1997; Schmahmann and Sherman, 1998; Karatekin et al., 2000; Neau et al., 2000; Bellebaum and Daum, 2007; Balsters et al., 2013; Wu et al., 2013; Rentiya et al., 2017), and language (Petersen et al., 1989; Desmond et al., 1998; Fulbright et al., 1999; Leggio et al., 2000; Lurito et al., 2000; Seger et al., 2000; Moretti et al., 2002; Xiang et al., 2003; Grönholm et al., 2005; Frings et al., 2006; Ben-Yehudah and Fiez, 2008; Rauschecker et al., 2008; Mariën et al., 2009, 2014; Stoodley and Schmahmann, 2009; Highnam and Bleile, 2011; Argyropoulos and Muggleton, 2013; Keren-Happuch et al., 2014). However, the nature of cerebellar contributions to these cognitive functions remains unclear. 
Working memory, the ability to temporarily store and manipulate information for complex cognitive activities (Baddeley, 1998), is perhaps one of the most studied cognitive function that engages cerebellum. Based on the theoretical framework of working memory by Baddeley and Hitch (1974), a central executive system with limited attentional capacity is served by two subsidiary storage systems: the phonological loop for verbal information and the visuospatial sketchpad for visual information. The phonological loop comprises a phonological store, which can hold memory traces for a few seconds before they fade, and an articulatory rehearsal process that can refresh the memory trace through active rehearsal, which is analogous to sub-vocal speech (Baddeley, 1992). Previous neuroimaging studies of verbal working memory suggest that regions in left inferior temporal/parietal regions are associated with the phonological store, and the left inferior frontal regions are associated with articulatory control process (Paulesu et al., 1993; Awh et al., 1996; Fiez et al., 1996). Based on the known neuroanatomy of cerebro-cerebellar pathways (Middleton and Strick, 1994, 1997, 2001) and the use of a phase-specific Sternberg task (Sternberg, 1966), Desmond et al. (1997) proposed a neuroanatomical model of two cerebrocerebellar circuits participating in the phonological loop: one connecting the frontal cortex to the superior cerebellum, providing the articulatory rehearsal process for phonological encoding, and the other connecting the temporal/parietal cortex to the inferior cerebellum, providing temporary maintenance of phonological information. This model was supported by subsequent functional neuroimaging studies (Chen and Desmond, 2005; Kirschen et al., 2010), cerebellar patient studies (Silveri et al., 1998; Ravizza et al., 2006; Chiricozzi et al., 2008; Kirschen et al., 2008; Peterburs et al., 2010), cerebellar transcranial magnetic stimulation (TMS; Desmond et al., 2005) and transcranial direct current stimulation (tDCS; Boehringer et al., 2013) investigations.

In the literature of motor control, forward models have been postulated as the basic computation provided by the cerebellum in order to control the musculoskeletal system, especially for rapid movements when sensory feedback delay is unavoidable (Wolpert and Miall, 1996; Wolpert et al., 1998). Forward models are essentially internal "neural" models that mimic the motor apparatus, which provide predictions of the sensory consequences of movements before feedback is available. Given the homogeneous cytoarchitecture of the cerebellar cortex, some investigators have argued that there is a common computational operation performed throughout the structure, with difference in function derived from the local input-output connections with the cerebral cortex (Ramnani, 2006; Ito, 2008; Strick et al., 2009; Bellebaum et al., 2012; Ishikawa et al., 2016). If the computational principles are indeed similar across the cerebellum, then our understanding of cerebellar function in sensorimotor control might be relevant to cerebellar involvement in verbal working memory. Therefore, in the current study, we propose the right cerebellum plays a predictive role, similar to forward models in motor control, that is beneficial for rapidly engaging the phonological loop in verbal working memory.
Given our cerebro-cerebellar model of phonological loop described earlier, we hypothesize that the cerebellum contributes to verbal working memory by generating two distinct predictions: (1) predictions of the articulatory trajectory based on the encoded verbal items, which may involve planning of movements of our jaw, tongue, lips, and larynx; and (2) predictions of the content in the phonological store, which may involve streaming a sequence of phonemes for sub-vocal rehearsal process. In a typical verbal working memory task, the success of correct verbal recall depends both on setting up an articulatory trajectory of the encoded verbal items as well as active rehearsal. Thus, we hypothesize that increased error rates in verbal working memory performance would occur if either the frontal/superior cerebellum articulatory prediction, or the parietal/inferior cerebellum phonological prediction, or both, were disrupted.

Direct evidence of disruption of cerebellar prediction in motor control has been observed using TMS, a brain stimulation technique that can temporarily interrupt function of the targeted area with high temporal specificity. Miall et al. (2007) tested the cerebellar forward model in a hand movement trajectory task by applying TMS to the cerebellum while participants made a rapid reaching movement toward a remembered target. This resulted in trajectory errors that could be explained by movements that were planned based on the hand position $138 \mathrm{~ms}$ ago. They suggested that the observed directional deviation was a result of a temporary loss of cerebellar predictive function, which caused the planning of reaching movement to be based on the previous (out of date) state of the arm.

Inspired by the Miall et al. (2007) results, we designed an analogous experiment to test our hypothesis that right cerebellum plays a predictive role in verbal working memory. We used TMS to briefly interfere with right cerebellar function as the participants covertly rehearsed a sequence of encoded letters. In order to generate articulatory trajectories with a known state over time, we used guided rehearsal, where a series of \# signs, each representing a letter of the encoded sequence, was presented on the screen one at a time to pace the subject's rehearsal of the letter sequence. This rehearsal process was interrupted by TMS, at which time the subject was immediately asked to report if a probe letter was the correct next-letter in the sequence. On half the trials the probe was the correct next letter, and on the other half the probe was either one letter earlier (early probe) or later (late probe) in the sequence. We predicted that, like the Miall et al. (2007) investigation, TMS would make the state estimation of the articulatory trajectory out of date (i.e., the forward models would be predicting a letter that was earlier in the trajectory instead of the correct next letter). Consequently, we predicted that cerebellar TMS would cause the participant to more likely judge an early probe as being in the correct position (more errors in early probe condition), a correct probe as being too late in the sequence (more errors in correct probe condition), and a late probe as (definitely) too late in the sequence (no or fewer errors). We used a control site in right occipital lobe to assess the specificity of cerebellar TMS effects. 


\section{MATERIALS AND METHODS}

\section{Participants}

A total of 23 (seven males, 16 females) healthy young adults, age $19-30$ (mean $=22.26$ years, $S D=2.649$ years), with educational attainment of at least 8 years, participated in the study. All participants were native English speakers with normal or corrected-to-normal vision, had no history of head trauma, seizure or a family history of epilepsy, stroke, neurological or psychiatric disorders, and were not taking anxiolytic, antidepressant, neuroleptic, or sedative medication at the time of the study. This study was carried out in accordance with the recommendations of Institutional Review Board of the Johns Hopkins School of Medicine with written informed consent from all subjects. All subjects gave written informed consent in accordance with the Declaration of Helsinki. The protocol was approved by the Institutional Review Board of the Johns Hopkins School of Medicine.

\section{Tasks}

Participants were asked to covertly encode an array of six letters presented on a screen in uppercase, which was then removed from the screen after $2 \mathrm{~s}$. Participants were instructed to read the letters in the order that they appeared (read from left to right, first row then second row). After a short delay (500 ms), 2-4 \# signs then appeared on the screen one at a time (400 ms for each \# sign with a $150 \mathrm{~ms}$ blank screen between \# signs), each representing a placeholder of a letter in the encoding array. On half of the trials, participants received paired $20 \mathrm{~Hz}$ TMS pulses $150 \mathrm{~ms}$ prior to the last \# sign, followed by a probe letter $(3 \mathrm{~s}$, presented in lowercase with a question mark. Participants were instructed to press button 1 for "yes" with their right index finger to indicate that the probe letter matches the next letter in the sequence, and to press 2 for "no" with their right middle finger if the probe letter does not match the next letter in the sequence

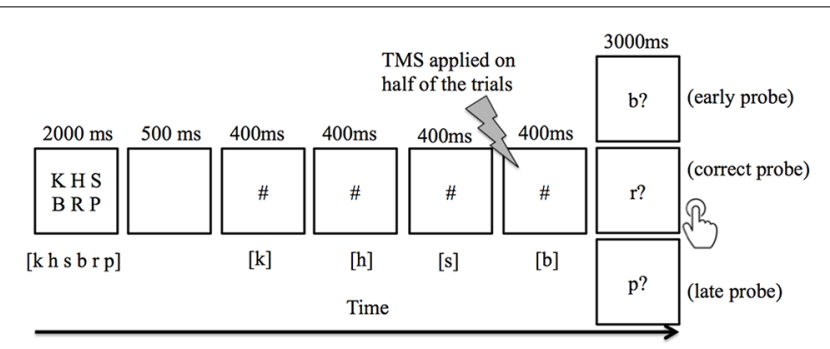

FIGURE 1 | An example of trial events progression in the verbal working memory task. Subjects were instructed to keep in mind six visually-presented letters, and to covertly rehearse the letters in sync with the appearance of \# symbols. Between each \# presentation, a 150 ms blank screen was included to visually separate the adjacent \# presentation. The letter(s) listed in [ ] indicates the correct content for rehearsal. When a probe letter appeared, the subject pressed button 1 to indicate that it matched the next letter in the sequence, or button 2 indicate that it did not match. The correct response for early probe and late probe conditions was the "non-match" button, whereas the "match" button was appropriate for the correct probe condition.

Double-pulse transcranial magnetic stimulation (TMS) was applied $150 \mathrm{~ms}$ prior to the last \# sign in half of the trials.
(Figure 1). In addition, they were instructed to respond as fast as possible without sacrificing accuracy. The next trial began after a fixed interval of 1,800 ms. Participants were given a short practice at the beginning of the experiment so that they could familiarize themselves with the task. During the task practice, feedback was given to indicate whether the participant's response was "Correct" or "Incorrect," and the accumulated percent accuracy was displayed. Feedback was not given during the actual TMS experiment.

A total of 80 trials were given for each session (cerebellum vs. occipital lobe stimulation, order counterbalanced). Eighty percent of these trials $(n=64)$ were longer trials with the probe letter appearing after the fourth \# sign (i.e., probe at the fifth position $=$ P5). The remaining $20 \%(n=16)$ containing either 2 or 3 \# signs (i.e., probe at the third or fourth position = P3 and P4) were discarded from analysis because a previous pilot study in our lab (unpublished) showed these shorter trials had a ceiling effect due to its short duration and thus lower working memory demands. However, the shorter trials were included to ensure that the timing of the probe letter was unpredictable. For both the longer trials (P5) and shorter trials (P3 and P4), there were an equal number of TMS and non-TMS trials as well as an equal number of match (i.e., correct probe) and non-match (i.e., early and late probe) trials.

\section{TMS Protocol}

Biphasic TMS paired-pulses were triggered at $20 \mathrm{~Hz}$ (i.e., $50 \mathrm{~ms}$ interpulse-interval) by E-Prime 2.0 standard software (Psychological Software Tool, Pittsburg, PA, USA) using a Magstim Rapid ${ }^{2}$ stimulator (Magstim Co., Whitland Dyfed, UK) that was connected to a $110-\mathrm{mm}$ diameter double cone coil. Motor threshold (MT) was determined for each participant as the minimal TMS intensity needed to evoke a visible muscle twitch in the right hand in 5 out of 10 trials upon stimulation of the left motor cortex. The coil was placed on the scalp with the handle held backward and with the coil current flowing in an upward direction at the juncture of the two loops of the coil. The optimal stimulation location and the orientation of the coil were marked on a fitting lycra swimming cap placed over participant's head to ensure consistent coil positioning.

For cerebellum stimulation, the double cone coil was centered at $1 \mathrm{~cm}$ below and $3 \mathrm{~cm}$ to the right of the inion. This coil geometry and position were found to be ideal for stimulating lateral cerebellar gray matter with low probability of passing through occipital cortex (Hardwick et al., 2014). However, in this position, we found that individual variations in skull shape created a gap between the scalp surface and the double cone coil to a varying degree in our participants. Previous studies have found that the scalp-coil distance directly influences the magnitude of stimulator output needed to reach MT (Kozel et al., 2000; McConnell et al., 2001; Stokes et al., 2007). Specifically, using a $70 \mathrm{~mm}$ figure-eight coil, Stokes et al. (2007) found for every $1 \mathrm{~mm}$ distance, an additional $\sim 2.8 \%$ of stimulator output was required to reach the same level of MT. However, to our knowledge, no studies have systematically manipulated the scalp-coil distance using a double-cone coil, which was designed for stimulating the deeper cortical areas typically at the depth 
of 3-4 cm from the scalp, as comparison to figure-eight coil at the depth of $2-2.5 \mathrm{~cm}$ ( $\mathrm{Lu}$ and Ueno, 2017). Therefore, before TMS stimulation of the cerebellum, we measured the MT for each participant at varying scalp-coil distances by placing custom-made moldable plastic separators ${ }^{1}$, measuring $3 \mathrm{~mm}$, $7 \mathrm{~mm}$, and $10 \mathrm{~mm}$ in thickness between the scalp surface and the coil. This resulted in four scalp-coil measurements: $0 \mathrm{~mm}$ (base level), $3 \mathrm{~mm}, 7 \mathrm{~mm}$, and $10 \mathrm{~mm}$. We then entered our measurements $(\mathrm{X}=$ separator thickness in $\mathrm{mm}, \mathrm{Y}=$ stimulator output needed to reach MT) into a linear regression equation to derive the slope and the constant. In order to measure the actual distance between the participant's scalp and the double cone coil for cerebellar TMS, we measured the scalp-coil distance using seven cylindrical wooden sticks in different diameters $(4.75 \mathrm{~mm}$, $6.43 \mathrm{~mm}, 8.2 \mathrm{~mm}, 9.75 \mathrm{~mm}, 11.25 \mathrm{~mm}, 12.5 \mathrm{~mm}, 15.04 \mathrm{~mm}$ ), after we positioned the participant in the TMS chair and placed the coil as close as possible to the scalp. The cylindrical stick that was the best fit between the scalp and coil was used to calculate the adjusted stimulator output using each individual's linear regression equation. The adjusted output number for scalp-coil distance was then multiplied by $110 \%$ to ensure excitability of the right cerebellum.

For the right occipital (control) region, the coil was centered at $7 \mathrm{~cm}$ above and $3 \mathrm{~cm}$ to the right of the inion. In this position, we did not experience any scalp-coil distance issues in all of our participants. Therefore, the stimulator output was directly set to $110 \%$ of MT. At this intensity, no participants reported phosphenes during the experiment. Finally, we note that the distance between the scalp and the targeted cortex is greater for cerebellum than for the occipital lobe, and consequently, the occipital lobe overall likely received more stimulation than the cerebellum.

\section{Data Analysis}

Error rate and reaction time (RT) were analyzed using SPSS version 24 (IBM Corp, Armonk, NY, USA). Differences in the percentage of error rate between TMS and non-TMS trials were calculated for each participant, separately for each cerebellar and occipital stimulation session. The same subtraction was performed for mean RT. Repeated-measures analysis of variances (ANOVAs) were then conducted on these differences to test for an interaction between stimulation sites (cerebellum vs. occipital lobe) and probe position (early, correct, late). Based on our hypothesis described in the "Introduction" section that cerebellar TMS would make the state estimation of the predicted sequence out of date, we predicted significantly higher error rates for early and correct probe, but not for the late probe. To test this a priori hypothesis, we conducted a planned comparison based on the predicted interaction between stimulation site (cerebellum $=+1$, control site $=-1$ ) and probe position (early probe $=+1$, correct probe $=+1$, late probe $=-2$ ), followed by three planned comparisons using paired $t$-tests to determine whether mean difference in error rate was significantly different between these two stimulation sites for each probe condition.

\footnotetext{
${ }^{1}$ http://InstaMorph.com
}

\section{RESULTS}

\section{TMS Coil-Scalp Distance and MT}

The mean MT (gap $=0 \mathrm{~mm}$ ) was $36.39 \%(\mathrm{SD}=5.813 \%)$. The mean slope for the linear regression was $0.709 \% / \mathrm{mm}$ $(\mathrm{SD}=0.23 \% / \mathrm{mm})$, which means an additional $0.709 \%$ of absolute simulator output was required for each $1 \mathrm{~mm}$ distance between the scalp and coil to reach the same level of MT excitability. For cerebellar stimulation, the average distance between coil and scalp was $9.464 \mathrm{~mm}(\mathrm{SD}=2.726 \mathrm{~mm})$. The average absolute stimulator output applied at the stimulation site was $46.91 \%(\mathrm{SD}=7.096 \%)$ for right cerebellum, and was $40.17 \%$ (SD $=6.415 \%$ ) for right occipital lobe. Additionally, no significant gender differences in TMS coil-scalp distance $\left(t_{(21)}=-0.769, p=0.450\right)$, slope $\left(t_{(21)}=1.068, p=0.298\right)$, and $\operatorname{MT}\left(t_{(21)}=1.158, p=0.26\right)$ were found.

\section{Accuracy Data}

Overall, participants made significantly more errors in TMS trials compared to non-TMS trials. For stimulation of the right cerebellum, the mean error rate for non-TMS trials was $13.5 \%$ $(\mathrm{SD}=8.6 \%)$, and increased to $23.9 \%(\mathrm{SD}=15.4 \%)$ for TMS trials. For stimulation of the right occipital lobe, the mean error rate for non-TMS trials was $12.8 \%$ ( $\mathrm{SD}=7.9 \%$ ), and increased to $16.2 \%$ $(\mathrm{SD}=12.2 \%)$ for TMS trials.

To examine whether the TMS effect on error rate is different between the two stimulation sites, we performed a repeated measure ANOVA with factors of stimulation site (cerebellum, occipital lobe) and trial type (TMS trial, non-TMS trial). This analysis yielded significant main effects of stimulation site $\left(F_{(1,22)}=5.719, p=0.026\right)$, trial type $\left(F_{(1,22)}=10.845, p=0.003\right)$, and a significant interaction effect $\left(F_{(1,22)}=6.333, p=0.020\right)$, with cerebellar stimulation resulting in higher error rate than occipital lobe on TMS trials (Figure 2).

To test our main a priori hypothesis that the early and correct probe would be more affected than the late probe by cerebellar TMS, we examined the error rate difference using a repeated measure ANOVA with factors of probe position

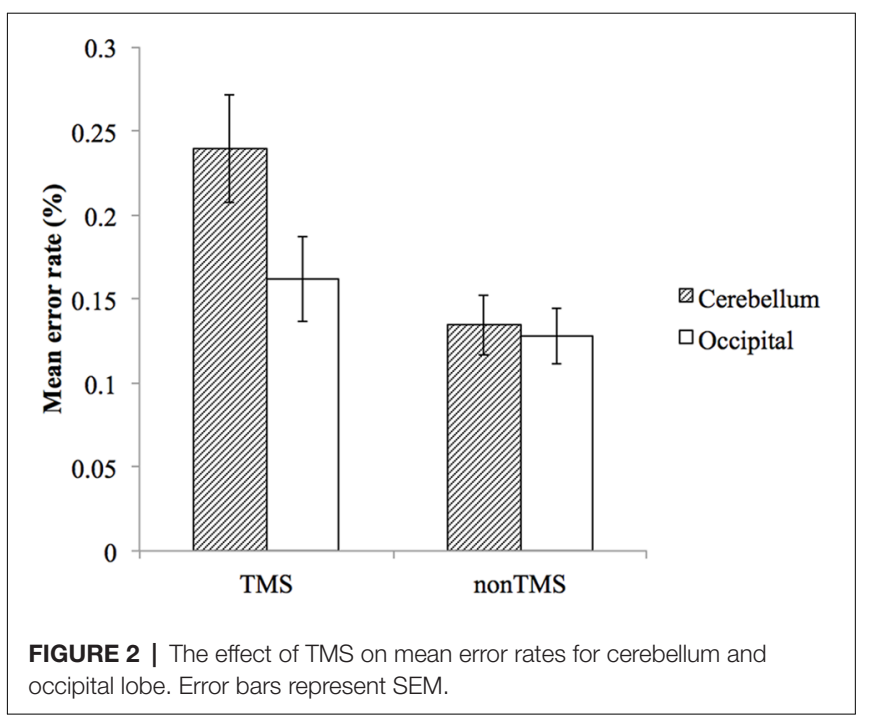




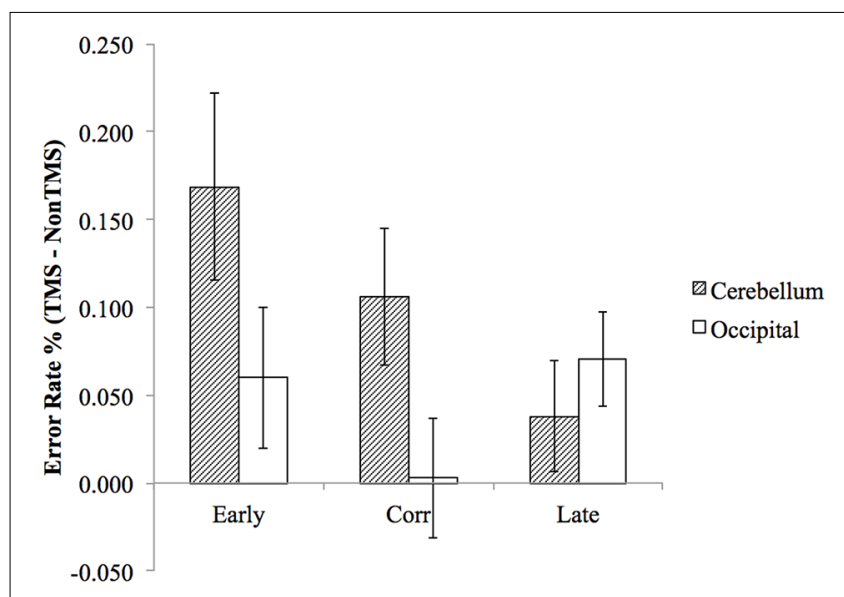

FIGURE 3 | The difference in (TMS minus non-TMS) error rates between cerebellum stimulation and occipital lobe stimulation was significantly greater for "early probe" and "correct probe" condition, but not for the "late probe" condition. Error bars represent SEM.

(early probe, correct probe, late probe) and stimulation site (cerebellum, occipital lobe). To perform this analysis, we first calculated the mean error rate difference by subtracting the error rate of non-TMS trial from TMS trials, and then entered them into the repeated measure ANOVA described above. Consistent with our hypothesis, the planned comparison of the interaction between probe positions and stimulation site confirmed that the cerebellar TMS relative to occipital TMS resulted in significantly higher error rate for early probe and correct probe, compared to late probe condition $\left(F_{(1,22)}=4.78, p=0.04\right)$. Direct paired $t$-tests were then conducted to assess the difference in error rate between cerebellar TMS vs. occipital TMS for early probe condition $\left(t_{(22)}=1.877, p=0.074\right)$, correct probe condition $\left(t_{(22)}=2.466, p=0.022\right)$, and late probe condition $\left(t_{(22)}=0.435\right.$, $p=0.795)$, as illustrated in Figure 3. The ANOVA also revealed a main effect for stimulation sites $\left(F_{(1,22)}=5.393, p=0.03\right)$, with higher error rate in cerebellar stimulation condition, a non-significant main effect for probe position $\left(F_{(2,44)}=1.645\right.$, $p=0.205)$, and an interaction effect that approached significance $\left(F_{(2,44)}=2.670, p=0.08\right)$.

\section{Reaction Time Data}

For the RT data, participants were slower on TMS trials (Mean $=750.888 \mathrm{~ms}, \mathrm{SD}=40.219 \mathrm{~ms})$ compared to non-TMS trials $($ Mean $=712.993 \mathrm{~ms}, \mathrm{SD}=30.647 \mathrm{~ms}$ ). For stimulation of the right cerebellum, the mean RT for non-TMS trials was $706.322 \mathrm{~ms}$ (SD = $130.218 \mathrm{~ms}$ ), and increased to $762.302 \mathrm{~ms}$ $(\mathrm{SD}=191.992 \mathrm{~ms})$ for TMS trials. For stimulation of the right occipital lobe, the mean RT for non-TMS trials was $719.664 \mathrm{~ms}$ (SD = $192.461 \mathrm{~ms}$ ), and increased to $739.474 \mathrm{~ms}$ ( $\mathrm{SD}=218.334 \mathrm{~ms}$ ) for TMS trials.

To examine whether the TMS effect on mean RT was different with respect to stimulation site, we performed a repeated measure ANOVA with factors of stimulation site (cerebellum, occipital lobe) and trial type (TMS trial, non-TMS trial). This analysis yielded a significant main effect of trial type $\left(F_{(1,22)}=5.805\right.$, $p=0.025)$ but not stimulation site $\left(F_{(1,22)}=0.029, p=0.867\right)$, and there was no significant interaction $\left(F_{(1,22)}=2.569, p=0.123\right)$. Thus, participants were significantly slower on TMS trials, but there was no difference in RT between TMS applied over cerebellum vs. occipital lobe.

We also examined whether the mean RT difference between TMS vs. non-TMS trials differed by probe position (early probe, correct probe, late probe) and stimulation site (cerebellum, occipital lobe). To perform this analysis, we first calculated the mean RT difference by subtracting the RT of non-TMS trials from TMS trials, and then entered them into a repeated measure ANOVA. This analysis yielded a significant main effect of probe position $\left(F_{(2,44)}=3.431, p=0.042\right)$, but not stimulation site $\left(F_{(1,22)}=0.879, p=0.359\right)$, and there was no significant interaction $\left(F_{(2,44)}=0.305, p=0.687\right)$. Upon examination of the mean RT difference (TMS RT-nonTMS RT), the correct probe has the greatest RT difference (Mean $=59.338 \mathrm{~ms}$, $\mathrm{SE}=17.673 \mathrm{~ms})$, followed by late $($ Mean $=37.122 \mathrm{~ms}$, $\mathrm{SE}=30.257 \mathrm{~ms})$, and finally the early probe $($ Mean $=-9.314 \mathrm{~ms}$, $\mathrm{SD}=20.223 \mathrm{~ms}$ ).

\section{DISCUSSION}

We found that TMS administration to the right cerebellum, applied during covert rehearsal of a remembered sequence of letters, resulted in an interference with participants' ability to identify whether a probe letter is in the correct position. Importantly, the pattern of results suggested that the response to the probe was based on out of date information regarding the next letter in the sequence. As a concrete example, in Figure 1, if TMS briefly causes the sequence prediction to be "frozen" at the letter B, then this letter b prediction will still be active when the probe letter is presented, and a probe letter of " $b$ " will seem to be correct, whereas the actual correct probe of " $r$ " will not, leading to errors on both the "early probe" and "correct probe" conditions. In contrast, "late probe" letters should still seem to be incorrect after TMS, and thus judgment of these letters should not be affected. The pattern of results depicted in Figure 3 supports this explanation and is consistent with our hypothesis that TMS pulses temporarily disrupt the function of the right cerebellum, resulting in prediction errors in the phonological loop. Our results therefore provide further evidence for cerebellar forward models in cognitive domains, in particular with respect to verbal working memory.

Our findings support the idea that cerebellar forward models contribute to verbal working memory by predicting upcoming verbal items in the phonological loop. In the motor control domain, the cerebellum is critical for predicting the outcome of an action before sensory feedback is available. These predictions can be compared with reafferent input. When they mismatch, an error signal is generated which allows rapid adjustments to the motor output as well as an update of the predictive model to refine future sensory predictions (Wolpert and Miall, 1996; Wolpert et al., 1998). Here, we presented evidence that the predictive capability of the cerebellum can be extended to verbal working memory. 
We proposed a cerebellar forward model that rapidly engages the phonological loop by computing an articulatory trajectory of the phonemes during the encoding phase. During the maintenance phase, the predicted output of the rehearsal process needs to be constantly compared to the content in the phonological store, which holds the correct sequence of phonemes kept in working memory. In previous studies, the encoding-related articulatory control process has been linked to right superior cerebellum via connection with Broca's area and premotor cortex, while the maintenance-related phonological loop has been linked to right inferior cerebellum via connection with left inferior parietal lobule (Chen and Desmond, 2005). In the current study, TMS was administered to the right cerebellum during the guided rehearsal process. Since cerebellar sub-regions cannot be clearly delineated with TMS techniques, the significantly higher error rate could be a result of a compromised predictive process in the phonological store, the articulatory control system, or both. Interestingly, a recent functional magnetic resonance imaging (fMRI) study found activity in the right posterolateral cerebellum correlated with the predictability of upcoming sentence content, and the same cerebellar cluster that is sensitive to linguistic predictability was recruited in a phonological task, but not in semantic or orthographic tasks (Lesage et al., 2017). These results are consistent with our current findings and are in line with the idea that cerebellum plays a predictive role in verbal working memory and in language comprehension through prediction of phonological information.

Although our results provide further evidence for a forward model account of the cerebellar role in verbal working memory, there is no consensus regarding the basic function that the cerebellum provides for cognition, and other accounts such as the timing hypothesis (Keele and Ivry, 1990; Tesche and Karhu, 2000; Ivry et al., 2002; Leggio et al., 2011), and the sequencing hypothesis (Tesche and Karhu, 2000; Leggio et al., 2011), have also been proposed. According to the timing hypothesis, the cerebellum is essential for the representation of temporal relationships. In our experiment, the probe occurs at the fifth position in a six-letter sequence on $80 \%$ of the trials. Therefore, it is possible that participants developed a temporal prediction of the occurrence of the probe stimulus, and that application of TMS disrupted the internal timing component, resulting in an increased error rate. However, under a timing hypothesis, we would expect all probe types to be equally affected by cerebellar TMS. Our data clearly showed that the error rate significantly increased in the early and correct probe conditions, but not in the late probe condition. Hence, the pattern of results may be better understood in the context of forward models. Another putative cerebellar function is sequence detection, which emphasizes the cerebellum's ability to detect and simulate repetitive sequence. It has been suggested that sequence detection is closely related to the predictive function characterized by forward models: the cerebellum creates internal models based on the sequence of events it detects (Leggio and Molinari, 2015). In verbal working memory, the "sequence" simulated by the internal model can be the content in the phonological store or the intended articulatory trajectory for rehearsal, which are respectively compared with the actual output of sub-vocal articulation or the actual trajectory of rehearsal. Therefore, the sequencing hypothesis is compatible with forward model explanations, and complements our findings of the functional role of cerebellum in verbal working memory.

Our data revealed a significant increase of RT in TMS trials compared to non-TMS trials. However, the RT difference was not significantly different between the stimulation sites (cerebellum vs. occipital lobe). In addition, the TMS effect on RT was not modulated by probe types (early, correct, late probes) between the two stimulation sites. This pattern of RT results, together with the significant TMS effects on accuracy, indicate that TMS interferes with the content in verbal working memory, rather than the speed of processing. These results are seemingly in conflict with a previous study showing cerebellar TMS resulted in an increase in RT during verbal working memory performance, but had no effect on accuracy (Desmond et al., 2005). However, a closer look of the task design in the previous study revealed that the TMS was administered immediately after encoding when the demand for preparation of articulatory trajectory is highest. On the other hand, in the current task, the TMS was administered closer to the end of the guided rehearsal phase when phonological store demand is highest. Given the known frontal/superior cerebellum circuit for articulatory preparation, and parietal/inferior cerebellum circuit for phonological store (Desmond et al., 1997, 2003, 2005), the TMS RT effect may likely reflect a compromised frontal/superior cerebellar articulatory control system, and the TMS accuracy effect may likely reflect a compromised parietal/inferior cerebellar phonological storage system. Taken together, the fact that we observed a TMS effect on accuracy (i.e., the content in working memory was affected), but not RT (i.e., processing speed was not affected), provide additional support that the right cerebellum plays a role in non-motor aspects of verbal working memory, and that a cerebellar forward model could explain the contribution of the cerebellum to non-motor cognitive functions, such as phonological storage.

In conclusion, our results are consistent with the idea that the right cerebellum supports verbal working memory by predicting upcoming verbal items in the phonological loop. It is assumed that a predictive process similar to forward models in motor control can be extended to non-motor cognitive functions such as verbal working memory, and the present study is consistent with other recent neuromodulation investigations supporting forward models in predicting verbal content (Lesage et al., 2012; Miall et al., 2016; D’Mello et al., 2017). Given the converging evidence from neuroimaging and anatomical studies, we speculate that: (1) the right superior cerebellum receives an "efference copy" of the articulatory command from Broca's area, from which it generates a predicted articulatory trajectory of the encoded phonemes; and (2) the right inferior cerebellum receives an "efference copy" of the refresh phonological store command from the temporal/inferior parietal lobe, from which it generates a phonological trajectory of phonemes for active rehearsal. These predictions would then feedback to their respective cortical areas 
for speedy and accurate processing of phonological information in verbal working memory.

\section{DATA AVAILABILITY}

The datasets generated for this study are available on request to the corresponding author.

\section{AUTHOR CONTRIBUTIONS}

JD conceived the presented idea and supervised the project. JD and Y-SS designed the experiment, analyzed the data and wrote

\section{REFERENCES}

Argyropoulos, G. P., and Muggleton, N. G. (2013). Effects of cerebellar stimulation on processing semantic associations. Cerebellum 12, 83-96. doi: 10.1007/s12311-012-0398-y

Awh, E., Jonides, J., Smith, E. E., Schumacher, E. H., Koeppe, R. A., and Katz, S. (1996). Dissociation of storage and rehearsal in verbal working memory: evidence from positron emission tomography. Psychol. Sci. 7, 25-31. doi: 10.1111/j.1467-9280.1996.tb00662.x

Baddeley, A. (1992). Working memory. Science 255, 556-559. doi: 10.1126/science. 1736359

Baddeley, A. (1998). Working memory. C. R. Acad. Sci. III 321, 167-173. doi: 10.1016/S0764-4469(97)89817-4

Baddeley, A. D., and Hitch, G. (1974). Working Memory. New York, NY: Academic Press.

Balsters, J. H., Whelan, C. D., Robertson, I. H., and Ramnani, N. (2013). Cerebellum and cognition: evidence for the encoding of higher order rules. Cereb. Cortex 23, 1433-1443. doi: 10.1093/cercor/bhs127

Bellebaum, C., and Daum, I. (2007). Cerebellar involvement in executive control. Cerebellum 6, 184-192. doi: 10.1080/14734220601169707

Bellebaum, C., Daum, I., and Suchan, B. (2012). Mechanisms of cerebellar contributions to cognition in humans. Wiley Interdiscip. Rev. Cogn. Sci. 3, 171-184. doi: 10.1002/wcs.1161

Ben-Yehudah, G., and Fiez, J. A. (2008). Impact of cerebellar lesions on reading and phonological processing. Ann. N Y Acad. Sci. 1145, 260-274. doi: 10.1196/annals.1416.015

Boehringer, A., Macher, K., Dukart, J., Villringer, A., and Pleger, B. (2013). Cerebellar transcranial direct current stimulation modulates verbal working memory. Brain Stimul. 6, 649-653. doi: 10.1016/j.brs.2012.10.001

Buckner, R. L. (2013). The cerebellum and cognitive function: 25 years of insight from anatomy and neuroimaging. Neuron 80, 807-815. doi: 10.1016/j.neuron. 2013.10.044

Chein, J. M., and Fiez, J. A. (2001). Dissociation of verbal working memory system components using a delayed serial recall task. Cereb. Cortex 11, 1003-1014. doi: $10.1093 /$ cercor/11.11.1003

Chen, S. H., and Desmond, J. E. (2005). Temporal dynamics of cerebro-cerebellar network recruitment during a cognitive task. Neuropsychologia 43, 1227-1237. doi: 10.1016/j.neuropsychologia.2004.12.015

Chiricozzi, F. R., Clausi, S., Molinari, M., and Leggio, M. G. (2008). Phonological short-term store impairment after cerebellar lesion: a single case study. Neuropsychologia 46, 1940-1953. doi: 10.1016/j.neuropsychologia.2008.01.024

Desmond, J. E., Chen, S. H., DeRosa, E., Pryor, M. R., Pfefferbaum, A., and Sullivan, E. V. (2003). Increased frontocerebellar activation in alcoholics during verbal working memory: an fMRI study. Neuroimage 19, 1510-1520. doi: 10.1016/s1053-8119(03)00102-2

Desmond, J. E., Chen, S. H., and Shieh, P. B. (2005). Cerebellar transcranial magnetic stimulation impairs verbal working memory. Ann. Neurol. 58, 553-560. doi: 10.1002/ana.20604

Desmond, J. E., Gabrieli, J. D. E., and Glover, G. H. (1998). Dissociation of frontal and cerebellar activity in a cognitive task: evidence for a distinction between selection and search. Neuroimage 7, 368-376. doi: 10.1006/nimg.1998.0340 the manuscript. Y-SS and YL carried out the experiment. Y-SS took the lead in writing the manuscript with support from JD.

\section{FUNDING}

This work was funded by the National Institutes of Health (NIH/NIMH; grant number R01MH104588 to JD) and was also supported by the Eunice Kennedy Shriver National Institute of Child Health and Human Development of the National Institutes of Health under Award Number U54HD079123. The content is solely the responsibility of the authors and does not necessarily represent the official views of the National Institutes of Health.

Desmond, J. E., Gabrieli, J. D., Wagner, A. D., Ginier, B. L., and Glover, G. H. (1997). Lobular patterns of cerebellar activation in verbal working-memory and finger-tapping tasks as revealed by functional MRI. J. Neurosci. 17, 9675-9685. doi: 10.1523/jneurosci.17-24-09675.1997

D'Mello, A. M., Turkeltaub, P. E., and Stoodley, C. J. (2017). Cerebellar tDCS modulates neural circuits during semantic prediction: a combined tDCS-fMRI study. J. Neurosci. 37, 1604-1613. doi: 10.1523/jneurosci.281816.2017

Durisko, C., and Fiez, J. A. (2010). Functional activation in the cerebellum during working memory and simple speech tasks. Cortex 46, 896-906. doi: 10.1016/j. cortex.2009.09.009

Fiez, J. A., Raife, E. A., Balota, D. A., Schwarz, J. P., Raichle, M. E., and Petersen, S. E. (1996). A positron emission tomography study of the short-term maintenance of verbal information. J. Neurosci. 16, 808-822. doi: 10.1523/jneurosci.16-02-00808.1996

Frings, M., Dimitrova, A., Schorn, C. F., Elles, H.-G., Hein-Kropp, C., Gizewski, E. R., et al. (2006). Cerebellar involvement in verb generation: an fMRI study. Neurosci. Lett. 409, 19-23. doi: 10.1016/j.neulet.2006. 08.058

Fulbright, R. K., Jenner, A. R., Mencl, W. E., Pugh, K. R., Shaywitz, B. A., Shaywitz, S. E., et al. (1999). The cerebellum's role in reading: a functional MR imaging study. AJNR Am. J. Neuroradiol. 20, 1925-1930.

Grafman, J., Litvan, I., Massaquoi, S., Stewart, M., Sirigu, A., and Hallett, M. (1992). Cognitive planning deficit in patients with cerebellar atrophy. Neurology 42, 1493-1496. doi: 10.1212/wnl.42.8.1493

Grönholm, P., Rinne, J. O., Vorobyev, V., and Laine, M. (2005). Naming of newly learned objects: a PET activation study. Cogn. Brain Res. 25, 359-371. doi: 10.1016/j.cogbrainres.2005.06.010

Hardwick, R. M., Lesage, E., and Miall, R. C. (2014). Cerebellar transcranial magnetic stimulation: the role of coil geometry and tissue depth. Brain Stimul. 7, 643-649. doi: 10.1016/j.brs.2014.04.009

Hayter, A. L., Langdon, D. W., and Ramnani, N. (2007). Cerebellar contributions to working memory. Neuroimage 36, 943-954. doi: 10.1016/j.neuroimage.2007. 03.011

Highnam, C. L., and Bleile, K. M. (2011). Language in the cerebellum. Am. J. Speech Lang. Pathol. 20, 337-347. doi: 10.1044/1058-0360(2011/ 10-0096)

Ishikawa, T., Tomatsu, S., Izawa, J., and Kakei, S. (2016). The cerebro-cerebellum: could it be loci of forward models? Neurosci. Res. 104, 72-79. doi: 10.1016/j. neures.2015.12.003

Ito, M. (2008). Control of mental activities by internal models in the cerebellum. Nat. Rev. Neurosci. 9, 304-313. doi: 10.1038/nrn2332

Ivry, R. B., Spencer, R. M., Zelaznik, H. N., and Diedrichsen, J. (2002). The cerebellum and event timing. Ann. N Y Acad. Sci. 978, 302-317. doi: 10.1111/j. 1749-6632.2002.tb07576.x

Justus, T., Ravizza, S. M., Fiez, J. A., and Ivry, R. B. (2005). Reduced phonological similarity effects in patients with damage to the cerebellum. Brain Lang. 95, 304-318. doi: 10.1016/j.bandl.2005.02.001

Karatekin, C., Lazareff, J. A., and Asarnow, R. F. (2000). Relevance of the cerebellar hemispheres for executive functions. Pediatr. Neurol. 22, 106-112. doi: 10.1016/s0887-8994(99)00128-9 
Keele, S. W., and Ivry, R. (1990). Does the cerebellum provide a common computation for diverse tasks? A timing hypothesis. Ann. N Y Acad. Sci. 608, 179-207; discussion 207-211. doi: 10.1111/j.1749-6632.1990.tb48897.x

Keren-Happuch, E., Chen, S. H. A., Ho, M. H. R., and Desmond, J. E. (2014). A meta-analysis of cerebellar contributions to higher cognition from PET and fMRI studies. Hum. Brain Mapp. 35, 593-615. doi: 10.1002/hbm.22194

Kirschen, M. P., Chen, S. H., and Desmond, J. E. (2010). Modality specific cerebrocerebellar activations in verbal working memory: an fMRI study. Behav. Neurol. 23, 51-63. doi: 10.3233/BEN-2010-0266

Kirschen, M. P., Davis-Ratner, M. S., Milner, M. W., Chen, S. H. A., SchraedleyDesmond, P., Fisher, P. G., et al. (2008). Verbal memory impairments in children after cerebellar tumor resection. Behav. Neurol. 20, 39-53. doi: 10.3233/BEN-2008-0216

Kozel, F. A., Nahas, Z., deBrux, C., Molloy, M., Lorberbaum, J. P., Bohning, D., et al. (2000). How coil-cortex distance relates to age, motor threshold and antidepressant response to repetitive transcranial magnetic stimulation. J. Neuropsychiatry Clin. Neurosci. 12, 376-384. doi: 10.1176/appi.neuropsych. 12.3 .376

Leggio, M. G., Chiricozzi, F. R., Clausi, S., Tedesco, A. M., and Molinari, M. (2011). The neuropsychological profile of cerebellar damage: the sequencing hypothesis. Cortex 47, 137-144. doi: 10.1016/j.cortex.2009.08.011

Leggio, M., and Molinari, M. (2015). Cerebellar sequencing: a trick for predicting the future. Cerebellum 14, 35-38. doi: 10.1007/s12311-014-0616-x

Leggio, M. G., Silveri, M. C., Petrosini, L., and Molinari, M. (2000). Phonological grouping is specifically affected in cerebellar patients: a verbal fluency study. J. Neurol. Neurosurg. Psychiatry 69, 102-106. doi: 10.1136/jnnp. 69.1 .102

Lesage, E., Hansen, P. C., and Miall, R. C. (2017). Right lateral cerebellum represents linguistic predictability. J. Neurosci. 37, 6231-6241. doi: 10.1523/jneurosci.3203-16.2017

Lesage, E., Morgan, B. E., Olson, A. C., Meyer, A. S., and Miall, R. C. (2012). Cerebellar rTMS disrupts predictive language processing. Curr. Biol. 22, R794-R795. doi: 10.1016/j.cub.2012.07.006

$\mathrm{Lu}, \mathrm{M}$., and Ueno, S. (2017). Comparison of the induced fields using different coil configurations during deep transcranial magnetic stimulation. PLoS One 12:e0178422. doi: 10.1371/journal.pone.0178422

Lurito, J. T., Kareken, D. A., Lowe, M. J., Chen, S. H., and Mathews, V. P. (2000). Comparison of rhyming and word generation with FMRI. Hum. Brain Mapp. 10, 99-106. doi: 10.1002/1097-0193(200007)10:3<99::aid-hbm10>3.0.co;2-q

Mariën, P., Ackermann, H., Adamaszek, M., Barwood, C. H., Beaton, A., Desmond, J., et al. (2014). Consensus paper: language and the cerebellum: an ongoing enigma. Cerebellum 13, 386-410. doi: 10.1007/s12311-0130540-5

Mariën, P., Baillieux, H., De Smet, H. J., Engelborghs, S., Wilssens, I., Paquier, P., et al. (2009). Cognitive, linguistic and affective disturbances following a right superior cerebellar artery infarction: a case study. Cortex 45, 527-536. doi: 10.1016/j.cortex.2007.12.010

Marvel, C. L., and Desmond, J. E. (2010). The contributions of cerebrocerebellar circuitry to executive verbal working memory. Cortex 46, 880-895. doi: 10.1016/j.cortex.2009.08.017

McConnell, K. A., Nahas, Z., Shastri, A., Lorberbaum, J. P., Kozel, F. A., Bohning, D. E., et al. (2001). The transcranial magnetic stimulation motor threshold depends on the distance from coil to underlying cortex: a replication in healthy adults comparing two methods of assessing the distance to cortex. Biol. Psychiatry 49, 454-459. doi: 10.1016/s0006-3223(00)01039-8

Miall, R. C., Antony, J., Goldsmith-Sumner, A., Harding, S. R., McGovern, C., and Winter, J. L. (2016). Modulation of linguistic prediction by TDCS of the right lateral cerebellum. Neuropsychologia 86, 103-109. doi: 10.1016/j. neuropsychologia.2016.04.022

Miall, R. C., Christensen, L. O., Cain, O., and Stanley, J. (2007). Disruption of state estimation in the human lateral cerebellum. PLoS Biol. 5:e316. doi: 10.1371/journal.pbio.0050316

Middleton, F. A., and Strick, P. L. (1994). Anatomical evidence for cerebellar and basal ganglia involvement in higher cognitive function. Science 266, 458-461. doi: $10.1126 /$ science. 7939688

Middleton, F. A., and Strick, P. L. (1997). Dentate output channels: motor and cognitive components. Prog. Brain Res. 114, 553-566. doi: 10.1016/s00796123(08)63386-5
Middleton, F. A., and Strick, P. L. (2001). Cerebellar projections to the prefrontal cortex of the primate. J. Neurosci. 21, 700-712. doi: 10.1523/jneurosci.21-0200700.2001

Moretti, R., Bava, A., Torre, P., Antonello, R. M., and Cazzato, G. (2002). Reading errors in patients with cerebellar vermis lesions. J. Neurol. 249, 461-468. doi: 10.1007/s004150200040

Neau, J. P., Arroyo-Anllo, E., Bonnaud, V., Ingrand, P., and Gil, R. (2000). Neuropsychological disturbances in cerebellar infarcts. Acta Neurol. Scand. 102, 363-370. doi: 10.1034/j.1600-0404.2000.102006363.x

Paulesu, E., Frith, C. D., and Frackowiak, R. S. (1993). The neural correlates of the verbal component of working memory. Nature 362, 342-345. doi: $10.1038 / 362342 \mathrm{a} 0$

Peterburs, J., Bellebaum, C., Koch, B., Schwarz, M., and Daum, I. (2010). Working memory and verbal fluency deficits following cerebellar lesions: relation to interindividual differences in patient variables. Cerebellum 9, 375-383. doi: 10.1007/s12311-010-0171-z

Peterburs, J., Cheng, D. T., and Desmond, J. E. (2016). The association between eye movements and cerebellar activation in a verbal working memory task. Cereb. Cortex 26, 3802-3813. doi: 10.1093/cercor/bhv187

Petersen, S. E., Fox, P. T., Posner, M. I., and Mintun, M. (1989). Positron emission tomographic studies of the processing of single words. J. Cogn. Neurosci. 1, 153-170. doi: 10.1162/jocn.1989.1.2.153

Ramnani, N. (2006). The primate cortico-cerebellar system: anatomy and function. Nat. Rev. Neurosci. 7, 511-522. doi: 10.1038/nrn1953

Rao, S. M., Bobholz, J. A., Hammeke, T. A., Rosen, A. C., Woodley, S. J., Cunningham, J. M., et al. (1997). Functional MRI evidence for subcortical participation in conceptual reasoning skills. Neuroreport 8, 1987-1993. doi: 10.1097/00001756-199705260-00038

Rauschecker, A. M., Pringle, A., and Watkins, K. E. (2008). Changes in neural activity associated with learning to articulate novel auditory pseudowords by covert repetition. Hum. Brain Mapp. 29, 1231-1242. doi: 10.1002/hbm. 20460

Ravizza, S. M., McCormick, C. A., Schlerf, J. E., Justus, T., Ivry, R. B., and Fiez, J. A. (2006). Cerebellar damage produces selective deficits in verbal working memory. Brain 129, 306-320. doi: 10.1093/brain/awh685

Rentiya, Z., Khan, N. S., Ergun, E., Ying, S. H., and Desmond, J. E. (2017). Distinct cerebellar regions related to motor and cognitive performance in SCA6 patients. Neuropsychologia 107, 25-30. doi: 10.1016/j.neuropsychologia. 2017.10.036

Schmahmann, J. D. (2019). The cerebellum and cognition. Neurosci. Lett. 688, 62-75. doi: 10.1016/j.neulet.2018.07.005

Schmahmann, J. D., and Sherman, J. C. (1998). The cerebellar cognitive affective syndrome. Brain 121, 561-579. doi: 10.1093/brain/121.4.561

Seger, C. A., Desmond, J. E., Glover, G. H., and Gabrieli, J. D. (2000). Functional magnetic resonance imaging evidence for right-hemisphere involvement in processing unusual semantic relationships. Neuropsychology 14, 361-369. doi: 10.1037/0894-4105.14.3.361

Silveri, M. C., Di Betta, A. M., Filippini, V., Leggio, M. G., and Molinari, M. (1998). Verbal short-term store-rehearsal system and the cerebellum. Evidence from a patient with a right cerebellar lesion. Brain 121, 2175-2187. doi: 10.1093/brain/121.11.2175

Sternberg, S. (1966). High-speed scanning in human memory. Science 153 652-654. doi: 10.1126/science.153.3736.652

Stokes, M. G., Chambers, C. D., Gould, I. C., English, T., McNaught, E., McDonald, O., et al. (2007). Distance-adjusted motor threshold for transcranial magnetic stimulation. Clin. Neurophysiol. 118, 1617-1625. doi: 10.1016/j. clinph.2007.04.004

Stoodley, C. J. (2012). The cerebellum and cognition: evidence from functional imaging studies. Cerebellum 11, 352-365. doi: 10.1007/s12311-0110260-7

Stoodley, C. J., Desmond, J. E., and Schmahmann, J. D. (2012). "Functional topography of human cerebellum revealed by functional neuroimaging studies," in Handbook of the Cerebellum and Cerebellar Disorders, eds M. Manto, D. L. Gruol, J. D. Schmahmann, N. Koibuchi and F. Rossi (New York, NY: Springer), 735-764.

Stoodley, C. J., and Schmahmann, J. D. (2009). The cerebellum and language: evidence from patients with cerebellar degeneration. Brain Lang. 110, 149-153. doi: 10.1016/j.bandl.2009.07.006 
Strick, P. L., Dum, R. P., and Fiez, J. A. (2009). Cerebellum and nonmotor function. Annu. Rev. Neurosci. 32, 413-434. doi: 10.1146/annurev.neuro.31. 060407.125606

Tesche, C. D., and Karhu, J. J. (2000). Anticipatory cerebellar responses during somatosensory omission in man. Hum. Brain Mapp. 9, 119-142. doi: 10.1002/(sici)1097-0193(200003)9:3<119::aid-hbm2>3.3.co;2-i

Wolpert, D. M., and Miall, R. C. (1996). Forward models for physiological motor control. Neural Netw. 9, 1265-1279. doi: 10.1016/s0893-6080(96) 00035-4

Wolpert, D. M., Miall, R. C., and Kawato, M. (1998). Internal models in the cerebellum. Trends Cogn. Sci. 2, 338-347. doi: 10.1016/S1364-6613(98) 01221-2

Wu, T., Liu, J., Hallett, M., Zheng, Z., and Chan, P. (2013). Cerebellum and integration of neural networks in dual-task processing. Neuroimage 65, 466-475. doi: 10.1016/j.neuroimage.2012.10.004
Xiang, H., Lin, C., Ma, X., Zhang, Z., Bower, J. M., Weng, X., et al. (2003). Involvement of the cerebellum in semantic discrimination: an fMRI study. Hum. Brain Mapp. 18, 208-214. doi: 10.1002/hbm.10095

Conflict of Interest Statement: The authors declare that the research was conducted in the absence of any commercial or financial relationships that could be construed as a potential conflict of interest.

Copyright (c) 2019 Sheu, Liang and Desmond. This is an open-access article distributed under the terms of the Creative Commons Attribution License (CC BY). The use, distribution or reproduction in other forums is permitted, provided the original author(s) and the copyright owner(s) are credited and that the original publication in this journal is cited, in accordance with accepted academic practice. No use, distribution or reproduction is permitted which does not comply with these terms. 